\title{
SCANNING ELECTRON MICROSCOPY OF THE EGGS OF THREE HUMAN SCHISTOSOMES
}

\author{
JoHN W. Ford and HARVEY D. BlanKesPOOR* \\ Mollusk Division, Museum of Zoology, University of Michigan, Ann Arbor, MI 48109, U.S.A.
}

(Received 21 February 1977; in revised form 27 April 1978)

\begin{abstract}
Ford J. W. and Blankespoor H. D. 1979. Scanning electron microscopy of the eggs of three human schistosomes. International Journal for Parasitology 9: 141-145. The surface of the eggs of Schistosoma haematobium, $S$. japonicum and $S$. mansoni, examined by scanning electron microscopy, are covered with microspines. The spines of $S$. mansoni and $S$. haematobium are essentially similar; however, in $S$. japonicum they are smaller and more densely distributed. A fibrous matrix, present on the surface of the eggs, is not host derived. This matrix may account for the stickiness of the eggs and the micospines may function to hold the matrix in place.
\end{abstract}

INDEX KEY WORDS: Schistosoma haematobium, Schistosoma japonicum, Schistosoma mansoni, schistosomiasis, scanning electron microscopy, microspines, egg shells, matrix.

\section{INTRODUCTION}

SEVERAL investigators have recently used the SEM to study the adult topography of the three species of Schistosoma causing schistosomiasis in man. Ilowever, little has been published on the larval stages: the miracidium, sporocyst and cercaria; even less has been done on the eggs.

Trematode egg shells are composed of sclerotin, a stable 'tanned' protein (Smyth \& Clegg, 1959). The presence of microspines covering the surface of schistosome eggs was first reported by Stenger, Warren \& Johnson (1967). Working with the transmission electron microscope (TEM), they observed 'microspicules' on Schistosoma mansoni eggs. Race, Michaels, Martin, Larsh \& Matthews (1969) and Race, Martin, Moore \& Larsh (1971), using both the TEM and the SEM, confirmed this observation. Hockley (1968) observed that similar microspines covered the surface of $S$. haematobium eggs. Schnitzer, Sodeman, Sodeman, Durkee \& Durkee (1971), using the TEM, reported microspines on $S$. japonicum eggs. These microspines, which cannot be removed by enzymatic digestion, are continuous with the main part of the shell and are probably composed of the same material (Hockley, 1968). Articles by Hockley (1968), Race et al. (1971) and Sakamoto \& Ishii (1976) contain both low and high magnification scanning electron micrographs of $S$. mansoni eggs.

The present study was undertaken to examine and compare the egg shells of the three human schistosomes, to augment the previous work done on the TEM and SEM, and to provide better topographical details.

*Present address: Hope College, Holland, MI 49423, U.S.A.

\section{MATERIALS AND METHODS}

Albino mice infected with $S$, japonicum and $S$. mansoni were maintained in the laboratory until patent; similarly, hamsters served as definitive hosts for $S$. haematobium. These materials were provided by the Medical Malacology Laboratory in the University of Michigan. To recover eggs, infected animals were killed, their intestines removed and slit lengthwise, cleaned of their lumen contents and then homogenized. The homogenate was filtered through a set of sieves; the eggs thus recovered were transferred 10 a small finger bowl. Bactohaemagglutination buffer (DIFCO Laboratories), $\mathrm{pH} 7 \cdot 3$, used throughout this procedure, retarded hatching. Eggs were fixed in $3-5 \%$ phosphate-buffered formalin, rinsed in buffer, dehydrated in increasing concentrations of ethanol, moved gradually into pure amyl acetate, and then dried at the critical point using carbon dioxide. Specimens were mounted on metal stubs and vacuum coated with $300-400 \AA$ of gold. In a separate experiment, gravid females of $S$. japonicum were recovered from mice by perfusion, fixed and then processed for the SEM in the same manner as the eggs. Just before being gold coated, they were broken open to expose the surface of eggs held in utero. Micrographs were taken with the Jeol-JSM-U3 scanning electron microscope. Magnifications on the order of $1000 \times$ were used to examine such topographical features as egg shape, spination and surface texture; high magnifications served to give details of microspines covering the shell surface.

\section{OBSERVATIONS}

The shape of the eggs of all three species varied from broadly-oval to quite elongate-oval and some showed pronounced bulging around the center. The prominent lateral spine on $S$. mansoni eggs (Fig. 1) and the terminal spine on $S$. haematobium eggs (Fig. 2) were obvious topographical features distinguishing the two species. The rudimentary 
lateral spine of $S$. japonicum was a variable feature even within individual strains: on some eggs it appeared as a definite nubby spine (Fig. 3), on others it was no more than a bump on the shell and many eggs appeared to lack this spine altogether. At low magnifications, the microspines gave the egg surface a fine-grained appearance.

An additional feature observed was the fibrous, net-like matrix clinging to the shell surface. On $S$. haematobium and $S$. mansoni eggs this material appeared thinly spread across the surface of the shell or occasionally in dense patches. However, on the $S$. japonicum eggs it formed a consistently dense coat making observations of the shell surface difficult. It seemed possible that this matrix was deposited on the eggs as a host response. To test this possibility, gravid $S$. japonicum worms, processed whole for viewing on the SEM, were broken open just prior to gold coating to reveal eggs held in utero. These eggs were found to be densely covered with the same fibrous matrix (Figs, $4 \& 9$ ).

At magnifications above $5000 \times$, microspines covering the surface of the eggs became the dominant morphological feature. Microspines of $S$. haematobium (Figs. $5 \& 6$ ) and $S$. mansoni (Figs. $7 \& 8$ ) appeared essentially similar: as short, blunt protuberances covering the entire surface of the egg shell and extending nearly to the tip of the large spine. The interspinous distance was not constant and the density of the microspines varied somewhat from egg to egg. Microspines on the $S$. japonicum eggs (Fig. 10) differed in that they were clearly much smaller and more closely spaced. Some $S$, japonicum eggs had small, irregular patches of shell free of microspines. Whether this was natural or the result of abrasion during the processing is not known; similar patches were not observed on the other species.

\section{DISCUSSION}

Two previously published articles (Hockley, 1968; Race et al., 1971) contained scanning electron micrographs of whole $S$. mansoni eggs and high mag nification micrographs of the surface detail. In both reports the eggs had collapsed and the surface detail showed evidence of distortion. Such effects are likely due to drying of the eggs by evaporation of the alcohol used for dehydration. Surface tensions developing during evaporation have been demonstrated to cause such gross distortion of surface detail, even after fixation, as to make this technique of little value in the preparation of biological specimens for the SEM (Porter, Kelley \& Andrews, 1972). This problem can be overcome by drying of the specimens using the critical point method.

Hockley (1968), using the TEM, compared the microspines of $S$. mansoni and $S$. haematobium eggs and found them to be essentially of the same length, base width and interspinous separation, but different in shape; the $S$. mansoni were pointed whereas the $S$. haematobium were more rounded. Schnitzer et al. (1971) examined the microspine tips of $S$. haematobium in utero and observed them to be naturally rounded. The present micrographs show the $S$. mansoni microspines to be as rounded as those of $S$. haematobium. This may possibly be due to abrasion of the points during processing, but it seems more likely that the pointed tips, if present, are not visible because of the top view provided by the SEM (compare to the sectional view of the TEM). The finding that the microspines of $S$. japonicum are smaller and more densely distributed agrees with Schnitzer et al. (1971) who estimated these to be about one-fourth as long and half as wide as those of the other two species.

It has long been known that $S$. japonicum eggs seem to be coated with a sticky substance to which debris adheres, making light microscopy of the egg surface difficult (Cort, 1919). We also noticed the tendency of these eggs to clump together and to adhere tenaciously to glassware. It is suggested that this adhesive property is probably the result of the fibrous coating on the eggs. The lesser stickiness among eggs of the other two species is the result of a similar coating present in lesser quantity. It appears that the microspines may function, in part, to hold this matrix in place. Additional research will be necessary to determine the origin of the matrix.

\section{SUMMARY}

1. Eggs of Schistosoma haematobium, S. japonicum and $S$. mansoni were examined by scanning electron microscopy.

2. Their shape was found to vary somewhat although the majority of all three species were oval (this is at variance with the generally accepted more rounded shape of $S$, japonicum).

3. Eggs of all three human species are shown to be covered with microspines; those of $S$. haematobium and $S$. mansoni are essentially similar, while those of $S$. japonicum are smaller and more densely packed across the surface of the shell.

4. Schistosoma japonicum eggs, observed in utero, were found covered with a net-like fibrous matrix, also present to a lesser extent on the other two spccics. This matrix may account for the sticky property of the eggs and the function of the microspines may be to hold the matrix in place.

Acknowledgements-The authors are indebted to Dr. Henry van der Schalie, University of Michigan, for his efforts in improving the manuscript, and Dr. W. C. Bigelow, SEM Laboratory, Department of Materials and Metallugrical Engineering at the University of Michigan, for use of the scanning electron microscopy facilities. 

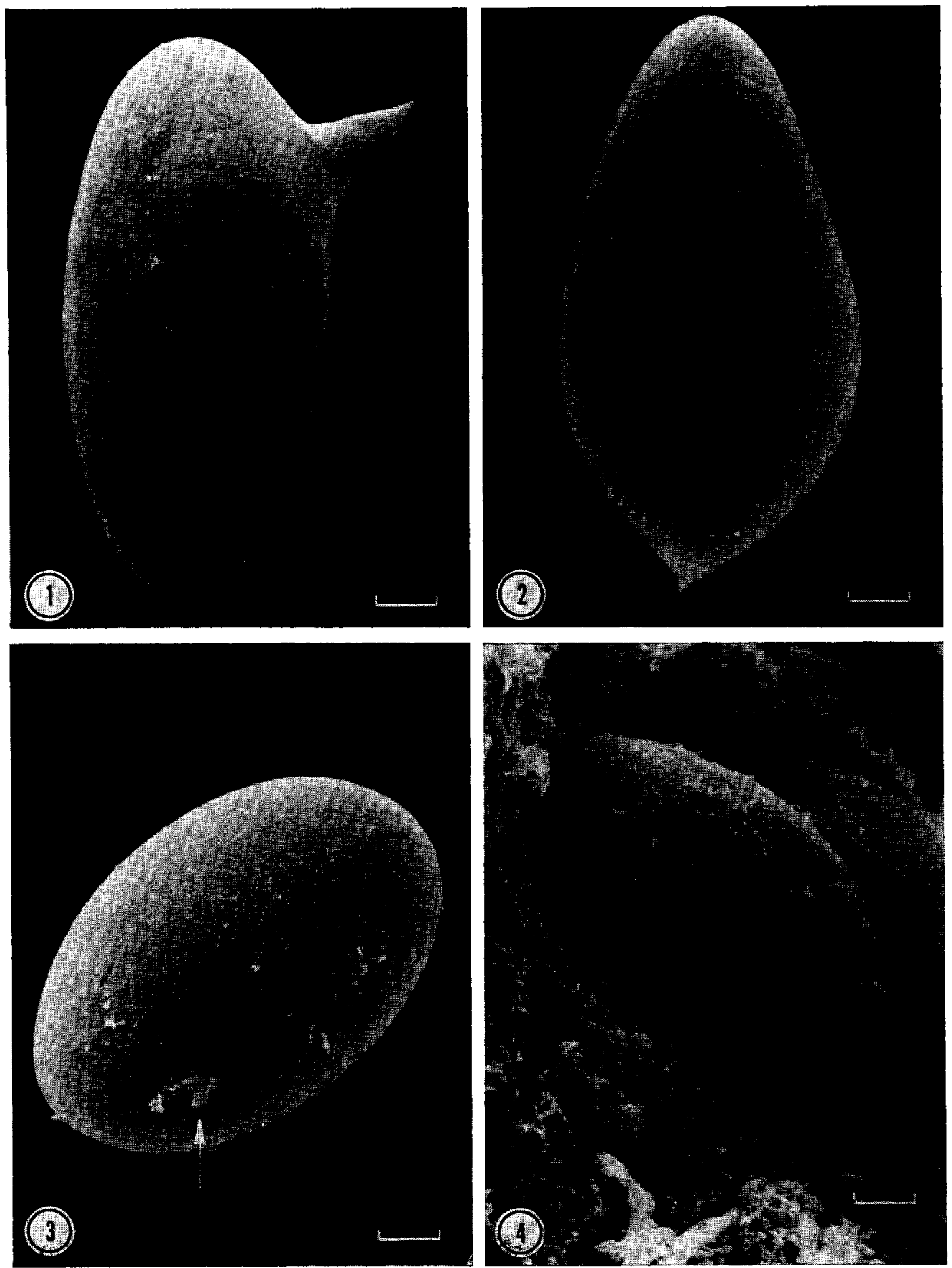

FIG. 1. Schistosoma mansoni egg (Scale $=10 \mu \mathrm{m})$.

FIG. 2. Schistosoma haematobium egg (Scale $=10 \mu \mathrm{m})$.

Frg. 3. Schistosoma japonicum egg showing a nubby lateral spine (arrow) (Scale $=8 \cdot 3 \mu \mathrm{m}$ ).

FIG, 4. $S$. japonicum egg in utero. The surface is coated with a fibrous matrix (Scale $=8 \cdot 3 \mu \mathrm{m}$ ). 

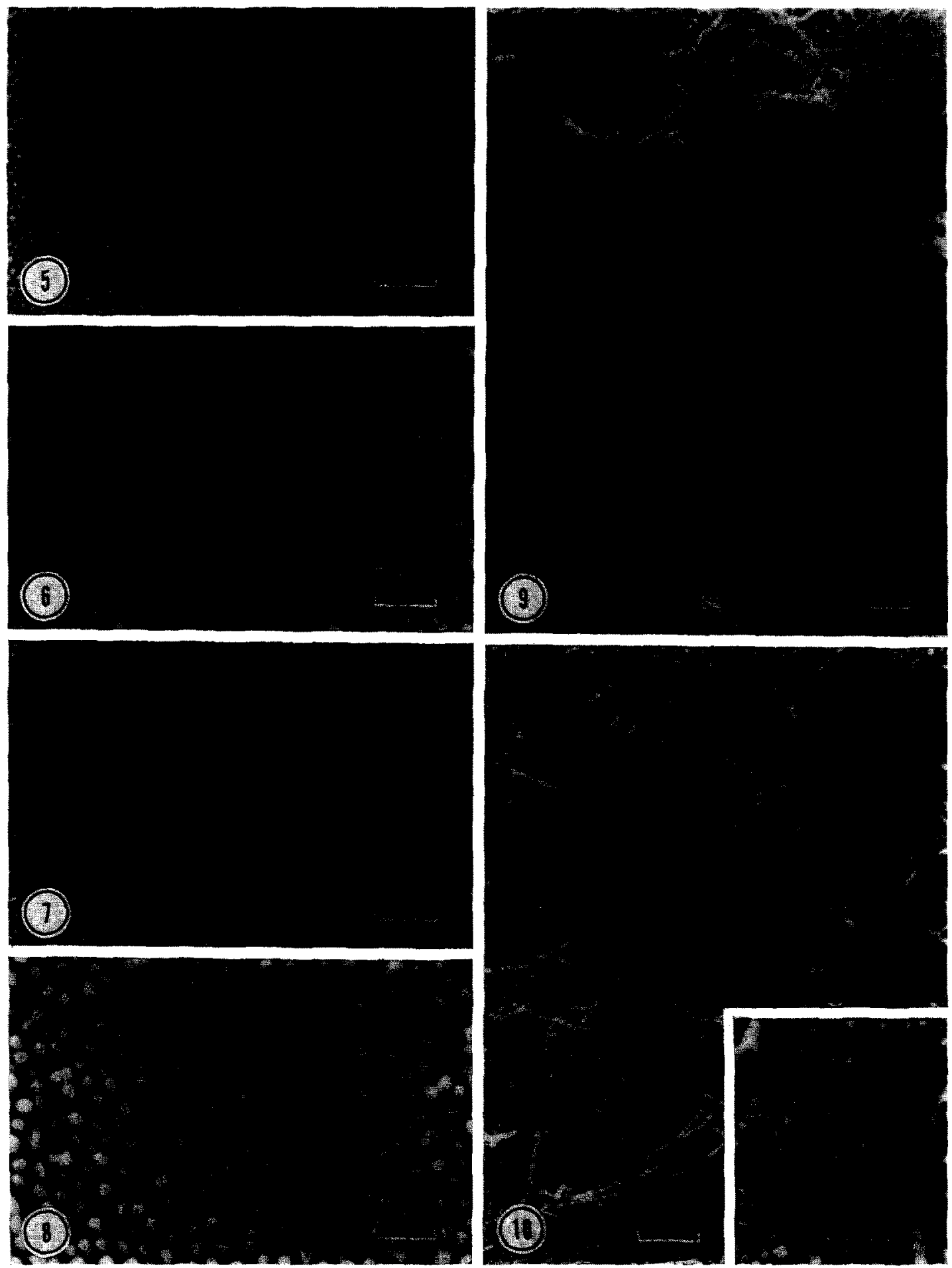

Fia. 5. Microspines on the surface of an $S$. haematobium $\mathrm{egg}$ (Scale $=0.7 \mu \mathrm{m})$.

Fic. 6. High magnification of the blunt microspines on an $S$. haematobium egg (Scale $=0.3 \mu \mathrm{m}$ ). Fig. 7. Microspines on an S. mansoni egg (Scale $=1.0 \mu \mathrm{m}$ ).

Fig. 8. High magnification of $S$. mansoni microspines (Scale $=0.3 \mu \mathrm{m}$ ).

Fig. 9. Detail of the fibrous matrix on an $S$. japonicum egg in utero (Scale $=1.0 \mu \mathrm{m}$ ).

FIG. 10. Microspines on an $S$. japonicum egg with remnants of the fibrous matrix (Scale $=1.0 \mu \mathrm{m}$ ). Insert (Scale $=0.3 \mu \mathrm{m}$ ). 


\section{REFERENCES}

Cort W. W. 1919. Notes on the eggs and miracidia of the human schistosomes. University of California Publications in Zoology 18: 509-519.

HoCkLey D. J. 1968. Small spines on the egg shells of Schistosoma Parasitology 58: 367-370.

Porter K. R., Kelley D. \& Andrews P. M. 1972. The preparation of cultured cells and soft tissues for scanning electron microscopy. Proceedings of the Fifth Annual Stereoscan Colloquium 1-19.

Race G. J., Michaels M., Martin J. H., Larsh, J. E., JR. \& Matthews J. L. 1969. Schistosoma mansoni eggs: an electron microscopic study of shell pores and microbarbs. Proceedings of the Society for Experimental Biology and Medicine 130: 990-992.

Race G J., Martin J. H., Moore, D. V. \& Larsh J. E., JR. 1971. Scanning and transmission electron microscopy of Schistosoma eggs, cercariae and adults. American Journal of Tropical Medicine and Hygiene 20: 914-924.

SaKamoto K. \& ISHII Y. 1976. Fine structure of schistosome egigs as seen through the scanning electron microscope. American Journal of Tropical Medicine and Hygiene 25: 841-844.

Schnitzer B., Sodeman T., Sonfman W. A., Durkff J. \& DURKeE T. 1971. Microspines on Schistosoma japonicum and S. haematobium egg shells. Parasitology 62: $385-387$.

SMYTH J. D. \& ClegG J. A. 1959. Egg-shell formation in trematodes and cestodes. Experimental Parasitology 8: 286-323.

Stenger R. J., Warken K. S. \& Johnsun E. A. 1967. An ultrastructural study of hepatic granulomas and schistosome egg shells in murine hepatosplenic schistosomiasis mansoni. Experimental and Molecular Pathology 8: 116-132. 